\title{
Rayleigh Instability-Driven Fragmentation of Ion Tracks
}

\author{
A. S. Khalil ${ }^{1}$ \\ ${ }^{1 .}$ Metallurgy and Mining Department, Tabbin Institute for Metallurgical Studies, Cairo, Egypt.
}

Ever since the success of invoking hydrodynamical analogues in diverse phenomena such as the famous water drop model of Bohr-Wheeler to explain the nuclear fission of uranium nuclei in 1939, the hydrodynamical interpretations in swift heavy ion (SHI) interaction with solids can generally be very rewarding [1]. It has been successfully used to explain different observations in this research field [2].

Indium Phosphide (InP) samples were prepared in standard TEM disks before irradiation using chemical thinning, in order to minimize any introduced preparation artifacts. The irradiation of samples by 200 $\mathrm{MeV}$ gold $(\mathrm{Au})$ ions was performed at room temperature using the 14 UD Pelletron accelerator of the Australian National University. By tilting the samples with respect to the illuminating electron beam to $\sim 30^{\circ}$ degrees in Philips 300CM TEM, we were able to reveal the lengthwise ion track morphology (i.e. along the ion trajectory and not only perpendicular to it, obtaining almost complete three dimensional information of the tracks rather than only observing the tracks cross sections). The ion tracks defined as trails of damage created by the Au ions penetrating InP were found to consist of chains of roughly equisized ( $\sim 10 \mathrm{~nm}$ in diameter) and equidistant nanospheres of damage as shown in figure 1 for a sample irradiated to a fluence of $5 \times 10^{10}$ ion $/ \mathrm{cm}^{2}$.

The TEM observations may point us to classical hydrodynamics analogues, particularly to the criteria for Rayleigh-Plateau instability for capillary-driven falling fluid columns and jets as water from a faucet breaks up into small droplets [3]. This phenomenon arises from a peculiar property of fluid cylinders, when the cylinder's length exceeds the cylinder's diameter by a factor of 3.13 it becomes energetically unstable. It will be driven towards a configuration that strives to minimize its net energy by amplification and propagation of perturbations whose wavelength exceeds the circumference of the cylinder. This instability leads to constriction, fragmentation and the eventual break-down into spheres. The resultant spherical configuration has less surface area compared to the initial cylinder of the same volume and thus is an energetically favored morphological end point as shown in figure 2 .

Calculations based on the thermal spike model for SHI irradiated InP $[4,5]$ showed that the temperatures along the ion path can be in excess of the melting point of $\operatorname{InP}\left(\mathrm{T}_{\mathrm{m}}=1335 \mathrm{~K}\right)$. Furthermore, the calculated lifetimes of melt-cylindrical zone around the ion path supports the existence of a transient liquid-like phase during track formation [4]. The Rayleigh mechanism is indeed plausible for ion tracks in InP from hydrodynamical point of view. Similar to the case of Rayleigh instability of heated metallic nanowires [6], ion tracks are characterized by large aspect ratio and large surface to volume ratio. Given the fact that high temperatures are present during track formation, there will be a need to minimize the free energy of the cylinder of melted material during the extreme short transitory period of its formation (time scales of $\sim 10^{-14} \mathrm{~s}$ ). Factors such as chemical potential minimization effects combined with possible defect diffusion on atomic scale and very rapid quench rates resulting from large radial thermal gradients will render the melt-cylinder along the ion path unstable $[7,8]$. It will be induced towards relaxation and final thermodynamical equilibrium state with the onset of Rayleigh instability, leading to fragmentation, pinching, and break-up into a chain of quenched-in nanoshperes of disordered material registered as an ion track in $\mathrm{InP}$. 


\section{References:}

[1] A. S. Khalil, PhD Thesis, Australian National University, Canberra (2007).

[2] D. Fink and L. Chadderton, Brazilian Journal of Physics 35 (2005), p. 735.

[3] Lord Rayleigh, Proceedings of the London Mathematical Society 10 (1878), p. 4.

[4] A. Komaru, W. Wesch, E. Wendler et al, Physical Review B 73 (2006), 184107.

[5] I. Amirkhanov, A. S. Khalil and I. Sarkhdov, Journal of Surface Investigation, X ray and Neutron Techniques, 7 (2013), p. 711.

[6] M. E. Toimil Molares, A. Balogh, T. W. Cornelius, R. Neumann and C. Trautmann, Applied Physics Letters 85 (2002), p. 5337.

[7] G. B. McFadden, S. R. Croeill B. T. Murray, The IMA Volumes in Mathematics and its Applications 53 (1993), p. 159.

[8] F. A. Nicholas and W. W. Mullins, Transactions of the American Institute of Mining, Metallurgical, and Petroleum Engineers 223 (1965), p. 1840.
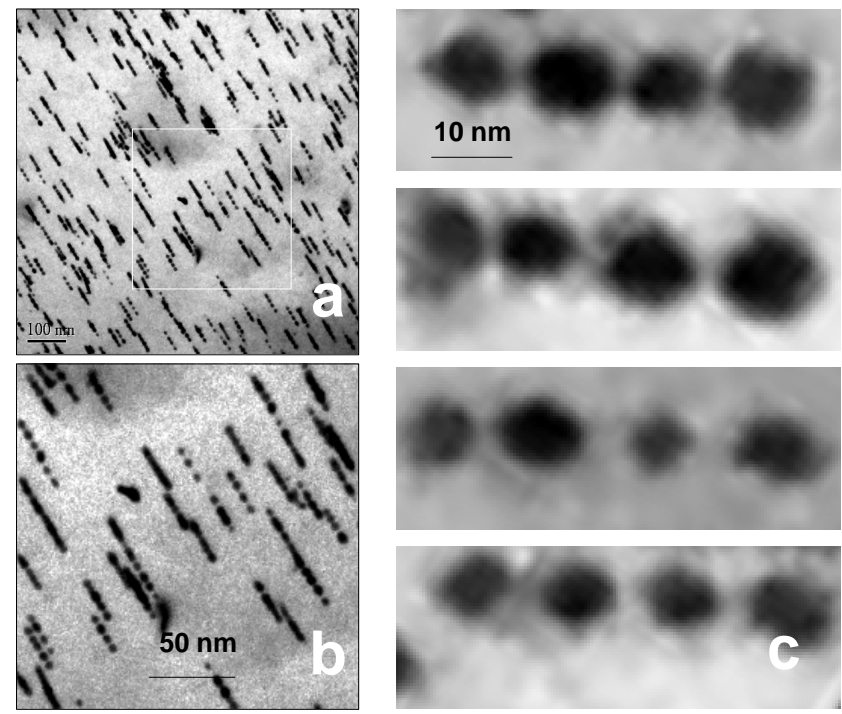

Figure 1. TEM micrograph of a field of ion tracks in InP (a), and in (b) an enlarged view of the area enclosed by the square in (a), and images of individual ion tracks revealing the peculiar morphology consisting of a chain of nanospheres of damage (c).
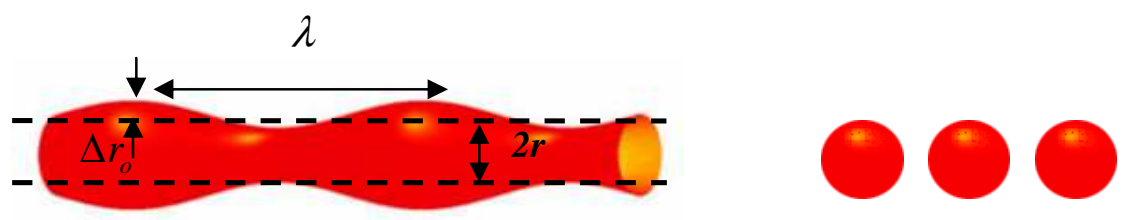

Figure 2. A schematic of the onset of Rayleigh instability in the form of perturbation with a characteristic wavelength $\lambda$ and amplitude $\Delta r_{o}$ along the axis of cylinder of $2 r$ diameter. In particular perturbation with $\lambda=8.89 \mathrm{r}$ will grow the fastest [8]. The cylinder is unstable and will eventually break up into a string of spheres. This instability might be responsible for the observed morphology of ion tracks in InP. 